Research paper

\title{
Intergranular pore space evolution in MX80 bentonite during a long-term experiment
}

\author{
Lukas M. Keller ${ }^{\text {a, } *}$, Lorenz Holzer ${ }^{\text {a }}$, Philippe Gasser ${ }^{\mathrm{b}}$, Rolf Erni ${ }^{\mathrm{c}}$, Marta D. Rossell ${ }^{\mathrm{c}}$ \\ a Zürich University of Applied Sciences, Winterthur, Switzerland \\ b Swiss Federal Institute of Technology, Centre for Imaging Science and Technology, Zürich, Switzerland \\ c EMPA, Materials Science and Technology, Electron Microscopy Center EMPA, CH-8600 Dübendorf, Switzerland
}

\section{A R T I C L E I N F O}

\section{Article history:}

Received 2 October 2013

Received in revised form 17 November 2014

Accepted 19 November 2014

Available online 4 December 2014

\section{Keywords:}

MX80 bentonite

Nanotomography

Gas transport

Percolation

ABM experiment

\begin{abstract}
A B S T R A C T
Focused ion beam nanotomography (FIB-nt) was applied to MX80 bentonite samples from the long-term Alternative Buffer Material (ABM) experiment in order to study the evolution of the intergranular pore space under similar condition that is supposed to prevail in repositories of nuclear waste. The applied high-resolution imaging method revealed the presence of two different types of pore filler. The first type is related to corrosion of iron and is represented by newly formed heavy minerals. Extensive formation of heavy minerals occurred only near the iron parts of the experimental set up. Based on comparison with other studies, the second filler type was interpreted as clay-gel that was likely formed during water uptake and swelling. A large fraction of the initial pore space was filled with such a clay gel. By attributing filled pores to the present open porosity, the initial intergranular porosity (radii $>10 \mathrm{~nm}$ ) of the starting material was in the range of $4.3-4.6 \mathrm{vol} . \%$, which was reduced to $<1 \mathrm{vol}$.\% during the experiment. A finite scaling approach was applied to the initial pore space (i.e. pores with radii $>10 \mathrm{~nm}$ ), which yielded percolation thresholds with critical porosities $\phi$ in the range of $3-19 \mathrm{vol} . \%$. Thus, the residual open porosity was far below the percolation threshold.

The initial porosity of one sample was above the percolation threshold, but also in this material percolation was restricted to one spatial direction. This indicated anisotropy with respect to percolation. The formation of a claygel and heavy minerals led to a decrease in intergranular porosity, which in turn affected connectivity of the pore network. Using results from pore-network modelling in combination with percolation theory illustrates that a minor reduction of porosity led to a substantial decrease in pore connectivity. Depending on water saturation within the observed intergranular pore space, air permeability decreases exponentially over three to four orders of magnitude within a narrow porosity range of about 1 vol.\%.

Based on observations and calculations, gas transport along the intergranular pore space of MX80 bentonite from the ABM experiment is not considered as a possible scenario and can reasonably be excluded.
\end{abstract}

(c) 2014 Elsevier B.V. All rights reserved.

\section{Introduction}

The present study investigates the pore structure of MX80 bentonite from the Alternative Buffer Material (ABM) experiment carried out at the Äspö Hard Rock Laboratory, Sweden. The aim of the experiment was to simulate conditions in a high-level radioactive waste depository. In order to study the degradation induced by the heat emission from radioactive waste, the buffer material was heated and water saturated simultaneously over an extended period of time ( $>1$ year). Reported dissolution and precipitation processes in the buffer material (e.g. Milodowski et al., 2007; Svensson et al., 2011) could have fundamental effects on the nano- to micron-scale structure of bentonites. Yet other modifications of the initial intergranular pore space are expected to occur during water saturation. The following scheme of intergranular

\footnotetext{
* Corresponding author. Tel.: +4158934 7778; fax: +4158935 7837.

URL: http://kelu(at)zhaw.ch (L.M. Keller).
}

pore evolution upon water uptake is widely accepted (Pusch et al. 1990; Pusch, 2001; Liu and Neretnieks, 2006). In the course of water saturation clay aggregates expand and after a sufficiently long time the intergranular pores will be filled by clay gel, which emerges from expanding aggregates. Thereby, the intergranular pore space is expected to be partly or entirely filled by the clay gel. Consequently, the objective of this study was to unravel the microstructural evolution of the intergranular pore space under similar conditions that are expected to prevail in nuclear waste repositories. At present a detailed knowledge of microstructural properties corresponding to variable bulk physical properties of MX80 bentonite is still lacking. In the context of the application as a back-fill material and the corresponding sealing behavior, microstructural investigations of the nanoscale pore space in bentonites using high-resolution imaging techniques are scarce, the work of Pusch (2001), Holzer et al. (2010) and Keller et al. (2014) being exceptions. At a larger scale of observation, other studies used X-ray computed microtomography to characterize the microstructure of bentonites 
(e.g. Tomioka et al., 2010). The microstructure of hydrating MX80 has been studied in detail indirectly using flow-thorough reaction cells and modelling of XRD patterns. This approach gives a quantification of the amount of water uptake in interlayers and pores (Perdrial and Warr, 2011).

During saturation and potential precipitation of new phases, intergranular porosity is expected to decrease and therefore it is important to know the smallest value of porosity (i.e. percolation threshold) and whether long-range percolation is still possible along the intergranular pore space. Such questions are important in the context of water and gas transport processes associated with the release of gases from iron corrosion in the repository. Bentonites are often thought to be impermeable to gas, because porosity that is relevant for gas transport (i.e. connected pores with radii $\geq 10 \mathrm{~nm}$ ) is low in this material. Gas migration experiments by Harrington and Horseman (2003) indicate that the original pore space of fully saturated MX-80 bentonites does not permit gas percolation at acceptable gas pressures. These experiments suggest the formation of pressure-induced pathways by some sort of microfracturing to allow gas flow. This indicates that the original volume fraction of larger pores is below the critical value for percolation. It is assumed that gas transport is restricted to larger pores (i.e. pores with radii $\geq 10 \mathrm{~nm}$ ) because gas flow along smaller pores is hampered due to capillary forces (Clayton and Hay, 1994; Marschall et al., 2005). However, the percolation threshold related to gas transport in bentonites is not known in detail since studies on pore connectivity in bentonites are largely absent.

Regarding bentonites, the main challenges of microstructural studies lie in the preparation of the samples so that they can be used for focused ion beam nanotomography (FIB-nt), which occurs under vacuum. Here we used state-of-the-art cryo sample preparation in combination with FIB-nt in order to obtain 3D reconstructions of the pore space and potential pore filler. The reconstructed pore microstructures were then analyzed with respect to percolation properties using the same procedure that was recently applied to Opalinus Clay (Keller et al., 2013a). In addition, changes in permeability due to modifications of the intergranular pore space are addressed and discussed.

In a recent study we investigated the pore structure of compacted and partially saturated MX-80 bentonite at different dry densities (Keller et al., 2014). FIB-nt was directly applied to laboratory prepared and high-pressure frozen samples in order to prevent drying artifacts (see Section 3.1.1). A further difference with the present study was the degree of water saturation of the sample material.

\section{Samples}

The investigated samples are from the experiment called alternative buffer material (ABM) (Eng et al., 2007). experimental set-up consisted of a borehole that is about $3 \mathrm{~m}$ deep and $300 \mathrm{~mm}$ in diameter (see Fig. 1). In the center of the hole an iron heater pipe was installed and the space between the pipe and the host rock was filled with cylindrical rings of different buffer materials that were stacked on top of each other. The use of an iron heater pipe allowed studying the interaction between iron and the buffer material. The experiment was equipped with a pipe system for artificial water saturation in order to speed up the saturation process. The examined samples are from an experiment, which ran for more than 2 years before retrieval. The buffer materials were heated (to $130{ }^{\circ} \mathrm{C}$ for $\sim 1$ year) and wetted from the start. With the heating pipe in the center of the experiment a temperature gradient evolved. Temperature measurements show that the temperature decreased to $<100{ }^{\circ} \mathrm{C}$ at distances $>60 \mathrm{~mm}$ away from the heater pipe. In the case of granulate "buffer blocks" a cage to hold the granulate in place around the central heating element was developed. For further details on the experiment, buffer ring manufacturing and characteristics of the buffer material the reader is referred to report IPR-07-15 (Eng et al., 2007).

After recovery of the samples, the buffer material was characterized in terms of bulk physical properties and mineralogical composition

\section{a) Experimental setup}

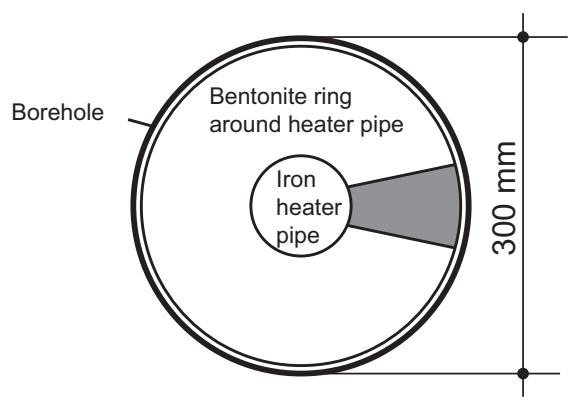

b) Sample localities FIB-nt

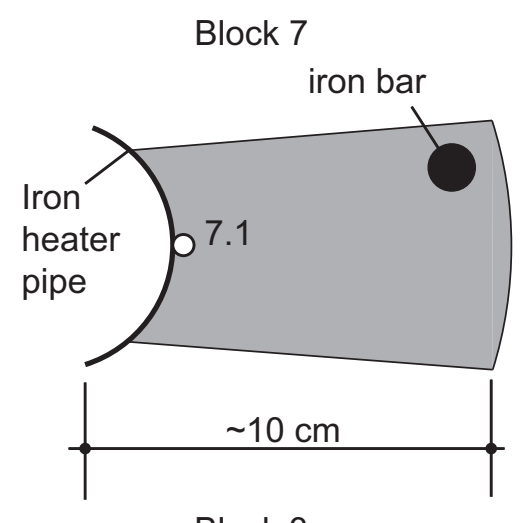

Block 3

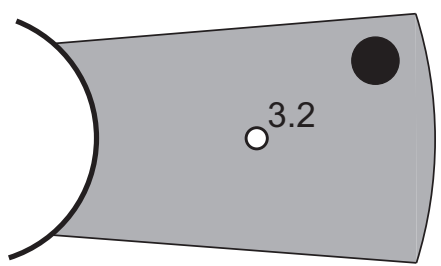

Fig. 1. Illustration of the set-up for ABM experiment. a) Cross-section of the borehole with a central iron heater pipe, around which rings of buffer material were installed. The gray slice marks the investigated sample material. b) Sketch of the sample material and the localities ( $=$ white circles) that were analyzed by FIB-nt.

(Svensson et al., 2011). The results relevant for the present study are summarized below. Carbonate dissolution was strongest proximal to the heater but occurred throughout the buffer ring since calcite peaks in XRD diffraction patterns were found only in the outermost parts of the ring (i.e. Block 2 MX80). The bulk compositions of the sampled bentonites were enriched in Fe when compared to pristine MX-80 reference material, indicating Fe transport into the bentonite.

In this study two samples were examined: i) a sample from the ABM-1 block 3 and ii) a sample from the ABM-1 block 7. Block 3 consists of MX80 granulate (i.e. Wyoming bentonite) and the one from block 7 consists of MX80 granulate with additional quartz. After retrieving, the samples were stored in a fridge under vacuum-packing to prevent them from drying.

\section{Methodology}

\subsection{Sample preparation}

Electron microscopy (FIB/SEM/TEM) requires drying of the samples prior to analysis. Conventional drying and/or freeze-drying of moist clay may cause preparation artifacts such as drying shrinkage (conventional 
drying) or ice formation during freeze-drying. Special methods, namely high-pressure freezing and subsequent freeze-drying were used to avoid these artifacts. The procedure for sample preparation in our study includes the following steps: Bentonite cores with a diameter of $5 \mathrm{~mm}$ were punched from the rather soft material with the help of punching tool. With the help of a scalpel we then cut slaps with a thickness of 200-300 $\mu \mathrm{m}$ from the core. Then, the slabs were frozen under high pressure (2100 bar) and within milliseconds by using the HPM 100 high-pressure freezing system. Freezing at high pressure occurs by the injection of pressurized liquid nitrogen. This treatment prevents the formation of ice-crystals and thus preserves the delicate framework of the pore space. Then, the vitrified water was sublimated under high vacuum using a system for freeze-drying (Holzer et al., 2010; Keller et al., 2011, 2013b). Details of high-pressure freezing techniques and their application for cryofixation are given by (Bachmann and Mayer, 1987). To stabilize the dry clay slabs, they were sandwiched between two $50 \mu \mathrm{m}$ thick glass disks which were glued together with epoxy. Cross-sections through the sandwiched samples were cut with a diamond saw. In order to analyze largely undisturbed material, FIB-nt and TEM were then applied in the center of the cross-sections (i.e. away from the rims of the sample which may be affected by mechanical truncation).

\subsubsection{On sample preparation artifacts}

The main challenges in studying bentonite microstructures lie in the preparation of samples so that they can be used for FIB-nt, which occurs under high vacuum and which can be overcome by cryofixation techniques which are used to investigate the microstructures of bentonites (Vali and Hesse, 1992; Bhuiyan, 2013). The most critical aspect of these cryofixation techniques is the cooling rate. If the cooling rate is too slow, the formation of ice crystals and phase separation can affect the original bentonite microstructure. To achieve the necessary cooling rates and to avoid this problem the sample thickness is limited. Here, we used a state of the art high-pressure freezing instrument that is able to freeze samples with a thickness of 200-300 $\mu \mathrm{m}$ within milliseconds so that they are completely vitrified. The frozen samples can then be analyzed either in frozen state by using a cryo-stage in the FIB/SEM or the vitrified intergranular pore water which can be sublimated by using a freeze-drying instrument prior to FIB-nt. The latter prevents artifacts induced by capillary forces, which could modify the delicate pore structure formed by individual clay grains.

One potential problem with the freeze-drying step is that the samples were defrosted under high vacuum (the sample was never exposed to higher temperatures than room temperatures) which leads to a loss of the interlayer water, which might alter the microstructure. Recently, Keller et al. (2014) performed a cryo-FIB-nt study to explore the microstructure of partly saturated MX-80 bentonite. Thereby, FIB-nt was applied directly to a high-pressure frozen bentonite sample by using a cryo-stage and cryo-transfer device to transport the sample between the instruments. During the study, the temperature of the frozen sample was increased in the sample chamber of the SEM from $-125^{\circ} \mathrm{C}$ to room temperature under the conditions of high-vacuum and the resulting effects on the microstructure were directly observed. In the frozen state, no microstructural modification was observed when saturated and compacted bentonite is exposed to high vacuum. As the clay grains are exposed to high vacuum there was no obvious loss of interlayer water at a pressure of $1.1 \mathrm{e}-6 \mathrm{mbar}$ and a sample temperature of $-125^{\circ} \mathrm{C}$. Any release of water can be detected because it affects the vacuum pressure of the instrument. Hence, we conclude that the exposure of swelling clay to high vacuum has no or only minor effects on the microstructure of the intergranular pore space.

In addition, after imaging in the frozen state the sample was slowly defrosted under high vacuum in order to sublimate free water (i.e. amorphous ice) (see Keller et al., 2014, their Figure (9)). During this process a few small open pores on the image surface were formed, indicating the presence of water filled pores. The release of free water was also confirmed by the breakdown of the vacuum caused by the degassing of water. Furthermore, the sublimation experiment showed the kind of microstructural artifacts that may be produced during clay sample drying. Cracks preferentially developed around larger grains are likely formed during the shrinkage of fine-grained matrix, which as a consequence separates from the surrounding (Keller et al., 2014). Apart from the development of cracks there are no other microstructural modification induced, indicating that the sublimation of free vitrified water under high vacuum does not significantly modify the bentonite in a way that renders the results of such studies to be meaningless. This finding applies to bentonite samples with high densities, however, in the case of diluted bentonite suspensions, sublimation of free water can certainly lead to significant changes in microstructure (Bhuiyan, 2013). As the ABM samples studied here have high packing densities, no artifacts caused by sublimation (freeze drying) were observed. As a result we consider the presented microstructures and extracted information of relevant properties to reflect the true behavior of the material under the application conditions used in the repository test.

\subsection{Focused ion beam nanotomography (FIB-nt)}

FIB-nt is achieved using dual beam FIB-SEM instruments, in which the ion and electron beams intersect under an angle of $52^{\circ}$ at a focal point on the sample surface. 3D information can be obtained by acquiring a sequence of cross sectional images by alternating FIB-slicing and SEM-imaging. The acquired series of images is spaced evenly through a region of the bulk specimen. The stack of those two-dimensional images can then be transformed into a three-dimensional representation of the microstructure in the sampled volume. The serial sectioning process begins by the milling of a wedge shaped trench in the sample. The 'front wall' of the trench is vertical (i.e., normal to the specimen surface) and it is chosen as the initial cross section, which is imaged by the electron beam (Holzer et al., 2004). After imaging, the ion beam is used to remove a layer of uniform thickness from the front wall, and thus the cross sectional plane advances to a predetermined distance through the sample volume. The next electron image is collected from the fresh cross-section. By repeating this alternating milling/imaging process, the cross section advances through the targeted volume, which results in a stack of 2D images. For this study, we used a Zeiss NVision 40 FIB/SEM instrument and important instrument setup parameters are listed below. SE and BSE image stacks were collected simultaneously to facilitate the segmentation process. For SEM imaging we used a low-acceleration voltage of $1.2 \mathrm{kV}$ and an aperture size of $30 \mu \mathrm{m}$. Ion gun milling current was $700 \mathrm{pA}$. The absolute size of the analyzed volumes, voxel size and bulk porosity are documented in Table 1.

\subsection{Image analysis}

The position of single SE images was aligned (in x- and y-coordinates) relative to the following image in the sequence (z-direction) by using an algorithm that is implemented in the free image processing software Fiji. BSE images were aligned according to the image coordinates obtained from the preceding alignment of the corresponding SE image stack. In this way, the image positions in both stacks from the same sample remain congruent. Then, the maximal overlapping area was cropped from the images of the aligned SE and BSE image stacks. Vertical stripes in the images are considered as artifacts from ion milling (i.e. the so-called waterfall effect). They were eliminated with a destriping filter. Then, a 3D background correction of the gray scale was applied in order to reduce systematic large-scale intensity variations, which are caused by shadowing effects related to the oblique imaging angle and to the subsidence of the image plane into the milled trench. Noise in the BSE images was reduced by application of an edge preserving 3D median filter of the Avizo software. The reconstruction of a 3D microstructure requires the segmentation of solid and pore phases in the images, i.e. the voxels representing particles, pores and pore fill have 
Table 1

Overview of properties of FIB-nt data sets.

\begin{tabular}{|c|c|c|c|c|c|}
\hline Sample locality & $\begin{array}{l}\text { Size } \\
{\left[\mu \mathrm{m}^{3}\right]}\end{array}$ & $\begin{array}{l}\text { Voxel size } \\
{[\mathrm{nm}]^{3}}\end{array}$ & Intergranular porosity & $\begin{array}{l}\text { Resolved pore radii } \\
{[\mathrm{nm}]}\end{array}$ & Figure \\
\hline 7.1 & 672.9 & $18^{3}$ & 0.046 & $\geq 16$ & Fig. 3 \\
\hline 3.2 & 196.5 & $10^{3}$ & 0.043 & $\geq 10$ & Fig. 5 \\
\hline
\end{tabular}

to be located in the images. For this purpose information of both congruent stacks (SE and BSE) can be combined with each other. The pore phase was segmented from the SE images, which show a higher gray level contrast between pores and surrounding material when compared to the BSE images. For the pore segmentation we used a multilevel threshold method based on the so-called Otsu thresholding (Otsu, 1979, see also Keller et al., 2011). Heavy minerals (see below) typically give a bright BSE signal in comparison to the surrounding clay matrix and thus they were segmented from the BSE images using gray level thresholding of the Avizo software. The information from segmentation was then combined into a single 3D volume where each of the phases (open and closed pores, clay matrix and heavy minerals) is marked with a characteristic label. For 3D visualization, we then used the Avizo software.

\subsection{Transmission electron microscopy}

For TEM imaging thin electron transparent lamellas are prepared from the clay rock material. For this purpose site-specific TEM foils are sampled with dimensions of around $10 \times 10 \times 0.1 \mu \mathrm{m}$ by using the focused ion beam (FIB) lift-out technique. TEM investigations were made using a JEOL 2200FS TEM/STEM equipped with a Gatan DigiScan system and operated at $200 \mathrm{kV}$.

\section{Results}

\subsection{Qualitative characterization of microstructure and porosity}

In this study we exclusively focus on intergranular porosity. Porosity was determined on the base of the segmented image stacks and corresponds to the ratio between void volume and the analyzed volume.

\subsubsection{FIB/SEM analysis of bentonite from block 7, locality 7.1}

The sample locality of the analyzed material from block 7 is directly adjacent to the contact with the central iron heater pipe (Fig. 1). Typical SE and BSE images from the FIB-stacks are presented in Fig. 2 for illustration and for qualitative description of the pore structure. In the SE images open pores appear as dark spots, which are marked with black arrows. However, only a very small amount of open pores are present in these samples.

SEM images reveal that some pores located between mineral grains (light gray) are filled with some material (Fig. 2). In the BSE images even two different types of pore filler can be distinguished based on the different gray scale levels (labeled as Pf1 and Pf2 in Figs. 2 and 3). However, the resolution in the BSE images is usually not sufficient for the detection of the very small open pores. The lack of resolution can be attributed to the relatively large excitation volume for the BSE signal. Hence, pores were detected from the SE-images and the volume fractions and size spectrum of different types of pore fills were distinguished on the basis of BSE-images.

A 3D reconstruction of the pore space and pore filler based on the entire SE- and BSE-stacks from FIB-nt is shown in Fig. 3. The porosity (radii $>16 \mathrm{~nm}$ ) (i.e. volume fraction of open pores) is $\sim 0.1 \mathrm{vol} . \%$. The volume fraction occupied by Pf1 is about 3.5 vol.\% and the one of Pf2 is about 1.0 vol.\%. Pf2 appears bright in BSE images and is thus interpreted as a heavy mineral. The origin and formation of the pore filler is discussed below.
Continuous pore and pore filler size distributions were determined (Münch and Holzer, 2008). The cumulative size distribution curves are shown in Fig. 4. The open pores are usually observed along the boundary and in corners of the original pore space. The majority of its pore radii are $<50 \mathrm{~nm}$. Radii of pore filler range between about 15 and $400 \mathrm{~nm}$. The slope of the cumulative PSD-curve in Fig. 4 increases steadily which documents that the pore volume fractions increase towards smaller radii.

\subsubsection{FIB/SEM analysis of bentonite from block 3, locality 3.2}

The sample locality of the analyzed material is in the center of the bulk bentonite ring, i.e. approximately $5 \mathrm{~cm}$ away from the iron heater pipe. Open intergranular pores and filled pores were segmented from the FIB/SEM-images in a similar way as described above for the sample from block 7. The pore filler that was segmented from the images can be attributed to type Pf1. As shown in Fig. 2b the bright pore fill (Pf2) is almost entirely absent in the sample from block 3 . This is also the case for the 3D-reconstruction of the pore microstructure, which is shown in Fig. 5. Only a very small amount of heavy minerals was observed in this volume.

In contrast to Block 7, the calculated pore size distribution of block 3 reveals a pore space that is characterized by much smaller pores (Fig. 4). The majority of pores have radii $<40 \mathrm{~nm}$. The porosity is around 0.5 vol.\% and the total fraction of pore filler is $3.6 \mathrm{vol}$.\%. There are some principle differences between the microstructures of block 3 and block 7 . The pores in block 3 are smaller than in block 7, which might indicate a higher degree of compaction.

\subsubsection{TEM-analysis of pore-fill}

Inspection of the TEM images depicted in Fig. 6 revealed that pore walls are bridged by delicate clay aggregates. The thickness of these bridges is around $10 \mathrm{~nm}$ and the gaps between the bridges are filled with another material. TEM diffraction patterns related to the material that fills the gaps were absent, which indicated that the material is poorly crystalline or even amorphous. Fig. 7 shows results of TEMEDX analysis. The brightness variation in the element distribution maps corresponds to the variation of the local concentrations. The material has a similar composition as the surrounding clay minerals but is slightly enriched in $\mathrm{Fe}, \mathrm{Ca}$ and $\mathrm{Si}$.

\section{Discussion}

\subsection{The nature of pore filler}

This study reports on the microstructure of MX- 80 granular bentonite from the ABM experiment. Initial bulk dry density for both samples was in the $\sim 1.4-1.5 \mathrm{~g} / \mathrm{cm}^{3}$ range, which during wetting increased to $\sim 1.9 \mathrm{~g} / \mathrm{cm}^{3}$ at almost full saturation. The sample from block 7 was taken directly at the central heating element and contains numerous heavy mineral grains (i.e. Pf2) (Fig. 2). In all probability, these grains were newly formed during the experiment and represent iron corrosion products related to the corrosion of the iron tube. Wersin et al. (2013) identified siderite and Fe-oxyhydroxides as corrosion products. The sample from block 3 is largely devoid of heavy minerals and was about $5 \mathrm{~cm}$ taken from the central iron tube. This is in support of the latter interpretation as the front, enriched in iron, extends about 1-2 mm into the clay (Wersin et al., 2013). 

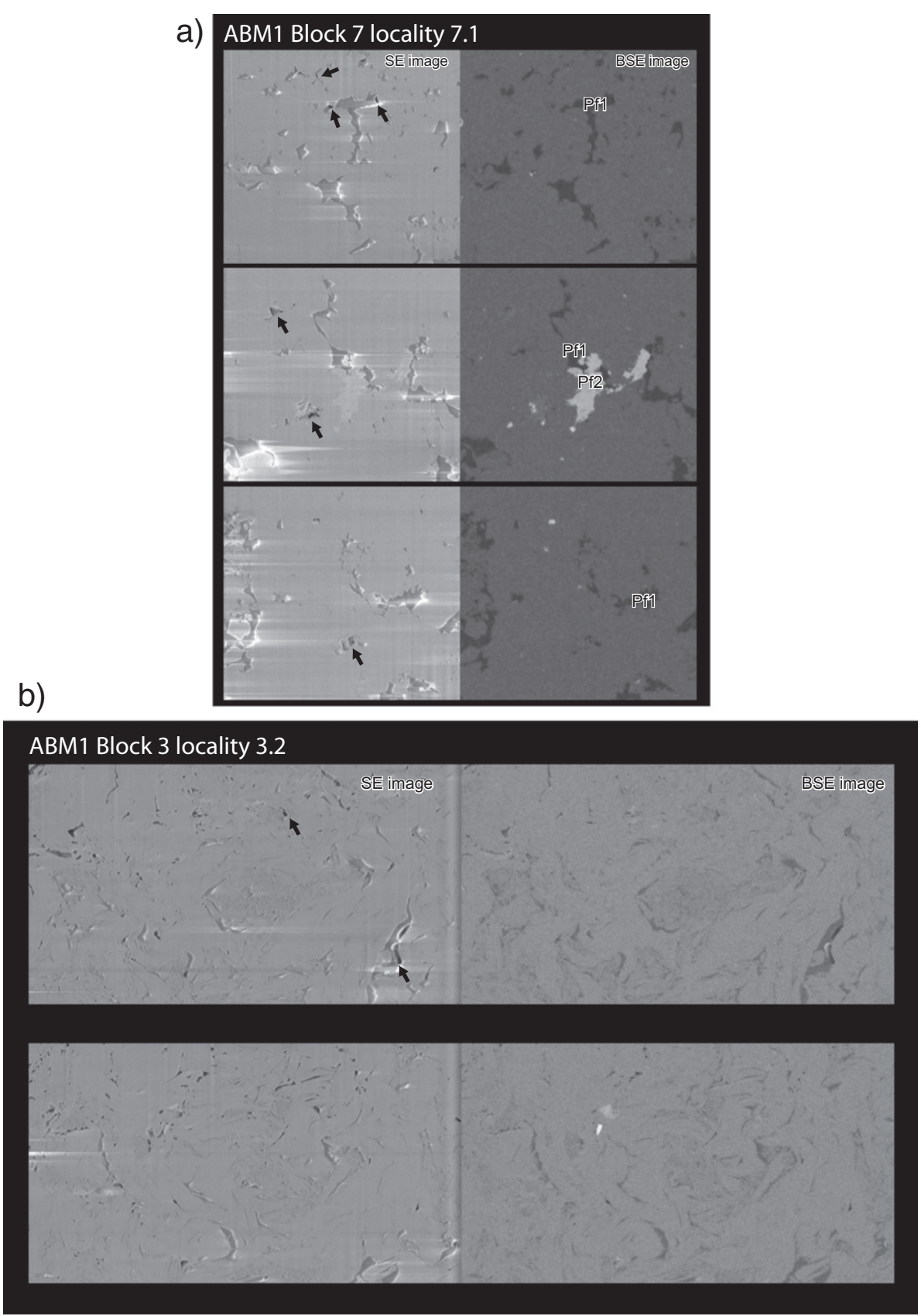

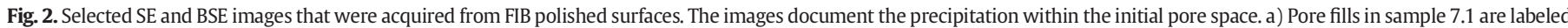

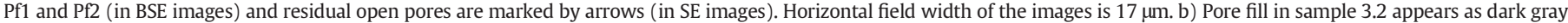
domain in BSE images. Residual open pores are marked by arrows in SE images. Horizontal field width of the images is $16 \mu \mathrm{m}$.

The interpretation of the other pore filler type (Pf1) is more difficult. In part these pores are bridged by filigree clay aggregate and the gaps between the bridges are filled with a material of poor crystallinity (Fig. 6). In addition, pore filler Pf1 is characterized by a "darker" BSE intensity when compared to the surrounding clay minerals, which indicates that its density is lower (Fig. 2). A material with similar properties was recently found in the course of a study, which investigated the microstructure of MX- 80 bentonite with dry densities similar to the one of the present study (Keller et al., 2014). In that study it was found that a clay gel fills the pores between mineral grains. This clay gel has different properties when compared to gels that form from clay suspension. The clay gels here have a higher clay concentration, lack free water and hence are more paste-like in nature. Its low yield characteristics under the force of gravity was confirmed by its sublimation behavior in the SEM, and taken to indicate the presence of a colloidal gel. In addition, the BSE intensity suggests that density of the paste-like material is lower, when compared to the surrounding clay minerals (Keller et al., 2014). Hence, Pf1 has similar material properties as a clay gel and is thus, interpreted as such. It is conceivable that a bentonite colloidal gel consisting of very small crystallites does not produce a conclusive TEM diffraction pattern. Hence, the absence of diffraction is in support of interpreting pore filler Pf1 as a colloidal gel. Assuming that pore filler Pf1 represents indeed a clay gel, its formation likely occurred during saturation, during which adsorption of water in the interlayer region causes the clay particles to separate and delaminate during swelling. After a sufficiently long time the initial intergranular pores will be filled with a clay-gel (Pusch et al. 1990, 2001; Keller et al., 2014). For the dry-density range under consideration, Keller et al. (2014) have shown that the extent of clay gel formation and associated degree of filling depend on the degree of saturation. Hence, it can 
a)

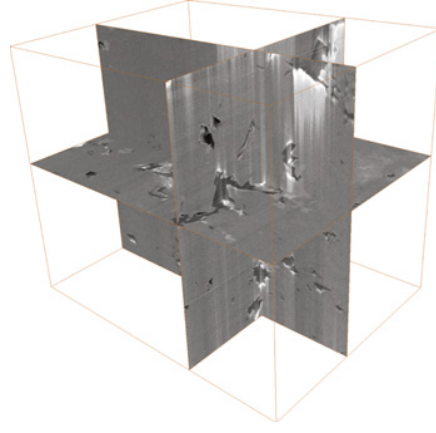

b)

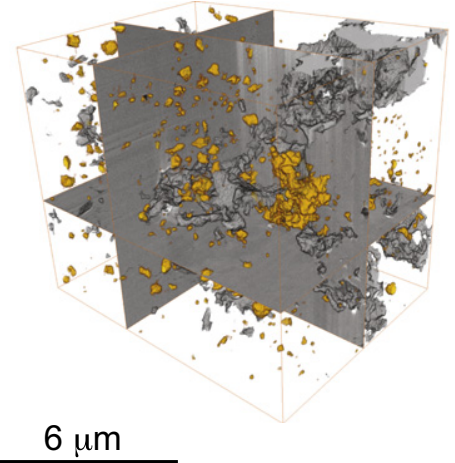

c)

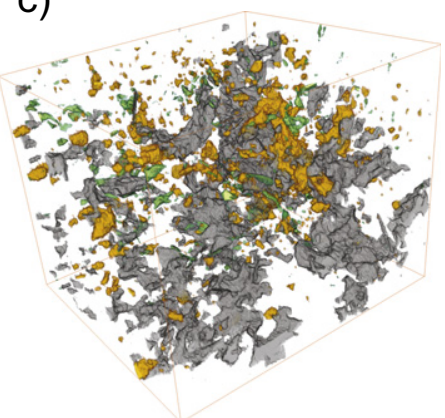

Open pores
Filled pores (Pf1)
Filled pores (Pf2)

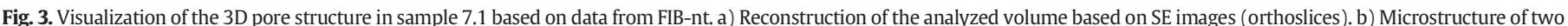

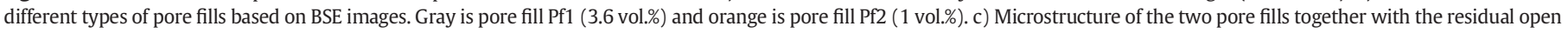
pores (= green, $<1$ vol.\%).

be expected that the initial porosity and associated transport properties are subject to major changes during saturation, which is now discussed in detail.

\subsection{Percolation threshold}

With increasing clay-gel content, the fraction of initial porosity decreases and the pore space is expected to change from large connected objects to isolated and unconnected features. During percolation transition, the probability of percolation $\lambda(\phi)$ changes in dependence of the present porosity $\phi$. Here the method introduced by Hilfer (1991) was used to calculate the probability of percolation (see also Keller et al., 2013a for more details). Calculated percolation probabilities $\lambda(\phi)$ in $\mathrm{x}$, $\mathrm{y}$ and $\mathrm{z}$ directions related to the initial porosity before clay-gel formation are displayed in Fig. 8. With increasing sample size L the width of the percolation transition decreases (Fig. 8). Note, that the transition width approaches zero if $L \rightarrow \infty$. Fig. 8 also shows that the porosity range does not cover the full transition from $\lambda_{\alpha}(\phi, L)=0$ to 1 for all sampled $L$. To improve the covered porosity range and to avoid the influence of local percolation fluctuations we applied the so-called smooth curves fit for the analysis of our data (see Pringle et al., 2009). These curves are subsequently used in the calculation of the percolation threshold (see below). In the case of the sample taken from block 7 (i.e. Fig. 8a to c), no anisotropy in connectivity is observed. On the contrary, the sample from block 3 has a higher connectivity in the $z$-direction and a lower connectivity along $\mathrm{x}$ - and $\mathrm{y}$-directions (Fig. $8 \mathrm{~d}$ to $\mathrm{f}$ ). It must be emphasized that the $\mathrm{x}$-axis in Fig. $8 \mathrm{f}$ has a different scale compared to Fig. 8a-e.

The critical porosity $\phi$ (i.e. percolation threshold) at which one can expect a percolating pore network is a crucial parameter. Here, we used two finite-size scaling schemes in combination with the results obtained from local percolation analysis to calculate the critical porosity (for details see Pringle et al., 2009; Keller et al., 2013a). The finite-size scaling relation $\phi_{\max }(L)-\phi_{c} \propto L^{-1 / \nu}$ was used to calculate the percolation threshold. In this relation, the effective percolation threshold is defined as the clay content $\phi_{\max }$ with maximum probability for the first occurrence of a percolating sample (Stauffer and Aharony, 1995). $\nu$ is the critical exponent, which according to percolation theory has a universal value depending only on the system dimension and in 3D, $\nu=0.88$ (Bunde and Havlin, 1995). Then, $\phi_{\max }$ corresponds to the a)

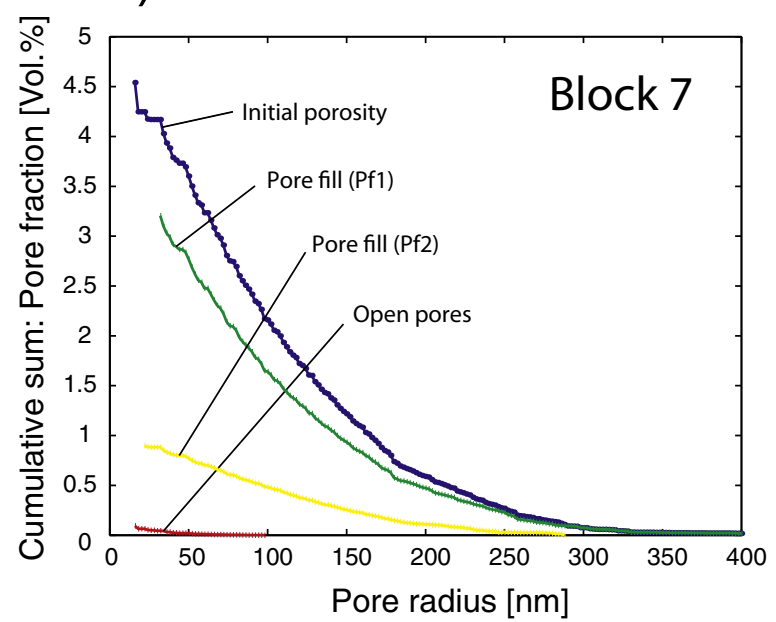

b)

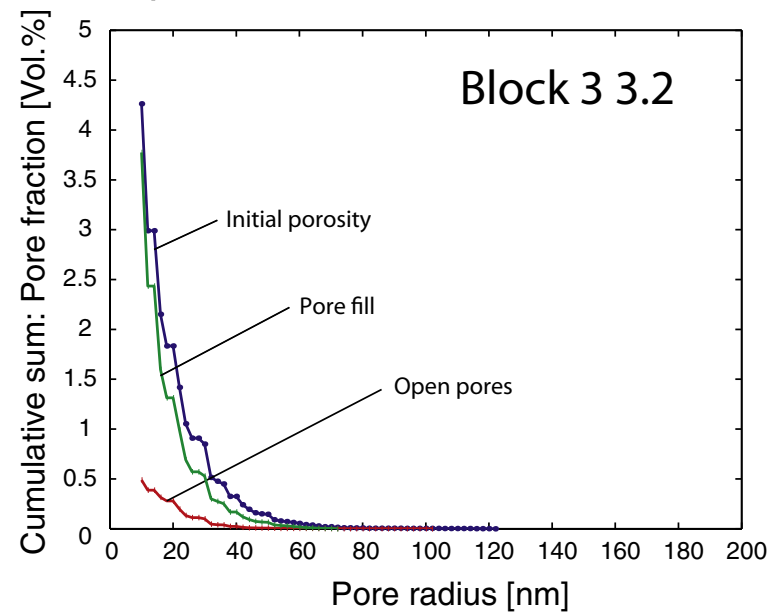

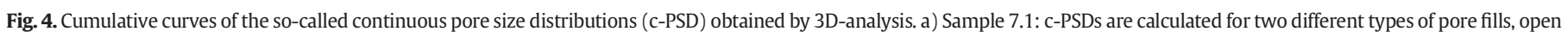
pores and initial porosity, which is the sum of open pores and filled pores. b) Sample 3.2: same as in Fig. 4a (without Pf2). 
a)

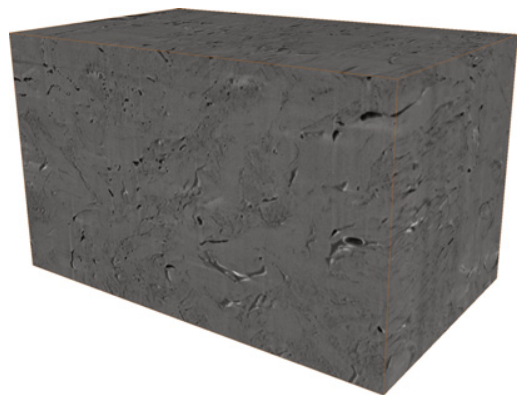

b)

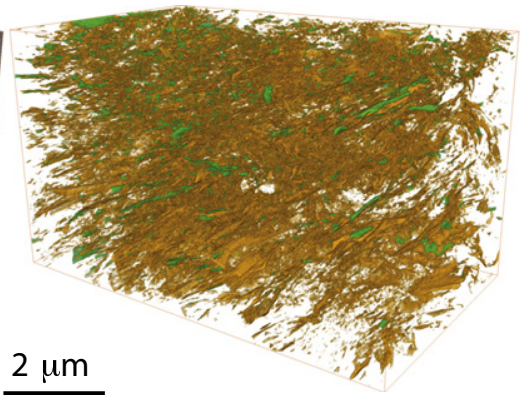

c)

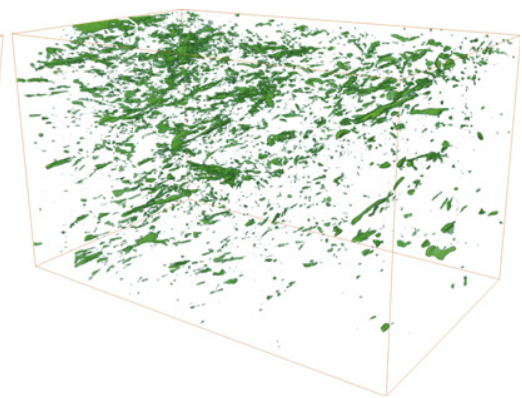

Open pores

Filled pores

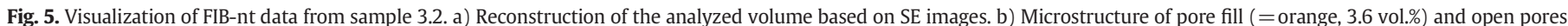
(=green, 0.5 vol.\%). c) Microstructure of residual open pores.

inflection point of $\lambda(\phi)$. Because $L^{-1 / v}$ decreases with increasing $L$, which implies that for $L \rightarrow \infty$, the value for the infinite size limit $\phi_{c}$ is given by the vertical axis intercept in a $\phi_{\max }$ versus $L^{-1 / v}$ plot. The same approach was used for the relation $\phi_{a v}(L)-\phi_{c} \propto L^{-1 / v}{ }_{c} \propto L^{-1 / v}$, where the effective percolation threshold $\phi_{a v}$ is the average porosity at which a percolating cluster appears for the first time (Stauffer and Aharony, 1995). Both finite scaling schemes give a similar picture of the direction dependent percolation thresholds, although $\phi_{a v}$ is slightly but consistently higher than $\phi_{\max }$. In the case of the sample taken from block 7 we have the relation $\phi_{c} \geq 0.07>\phi$ for all transport directions (Fig. 9a,b). Thus, the initial porosity (i.e. 0.046) is clearly below the percolation thresholds. In the case of the sample from block 3 we have $\phi_{c} \geq 0.09>\phi$ for the $\mathrm{x}$ - and $\mathrm{y}$-directions but for the $\mathrm{z}$-direction we obtain $\phi_{c} \geq 0.03<\phi$ (Fig. 9c,d). These results reveal a significant anisotropy with respect to the percolation thresholds, where the initial porosity of 0.043 exceeds the percolation threshold in the z-direction, but not in the $\mathrm{x}$ - and $\mathrm{y}$-directions. This is in agreement with the finding that only the two z-planes of the total analyzed volume are connected by a continuous network of pores.

\subsection{Permeability changes}

The formation of clay-gel and the precipitation of iron corrosion products along the initial pore space are expected to lead to changes in the gas transport properties of the bentonite seal. This effect is studied for the sample from block 3, in which the original porosity with radii $>10 \mathrm{~nm}(\phi=0.043)$ lies above the percolation threshold $\left(\phi_{c}=0.02-0.03\right)$ in the z-direction. We are interested in changes of gas permeability if $\phi$ approaches $\phi_{c}$ from above (i.e. $\phi_{\text {initial }}>\phi_{c}$, but $\phi$ decreases due to clay-gel formation). In terms of continuum percolation, gas permeability depends on the air filled porosity $\varepsilon$, which in turn depends on water saturation $S$. Therefore the air filled porosity may be given as $\varepsilon=(1-\mathrm{S}) \phi$. Water is the wetting fluid, which is retained in angular corners due to capillary forces. In this way it allows simultaneous flow of the two phases (i.e. gas and liquid). As a consequence, the critical volume fraction $\varepsilon_{c}$ for air percolation in a moist environment is smaller than the "dry" $\phi_{c}$. The air permeability $k_{a}$ can then be given as (Hunt, 2005):

$k_{a}=k_{o}\left[\frac{\varepsilon-\varepsilon_{c}}{\phi-\varepsilon_{c}}\right]^{t}$

with $\mathrm{t}=1.88$ in $3 \mathrm{D}$ and $k_{0}$ represents the value of air permeability $\left(k_{a}\right)$ in a completely dry medium. Intrinsic permeability is a flow property that characterizes transport of fluid phase (liquid or gas) through a connected pore structure. It is regarded as a property of the pore structure, which is independent on the characteristics of the fluid. Single-phase permeability is also referred to as the absolute permeability. In order to obtain a realistic value for the absolute permeability in the pore structure of the sample from block 3 we used the network simulator
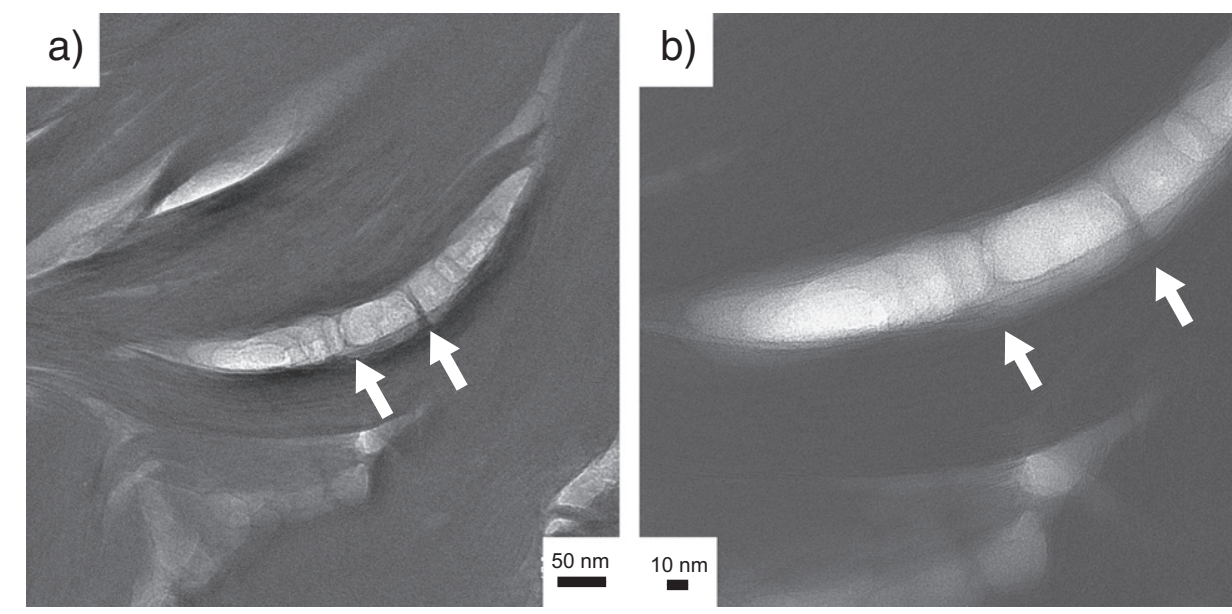

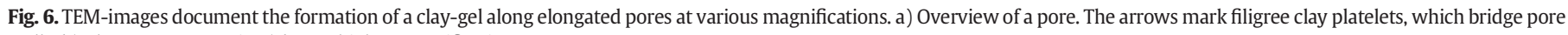
walls. b) The same pore as in a) but at higher magnifications. 


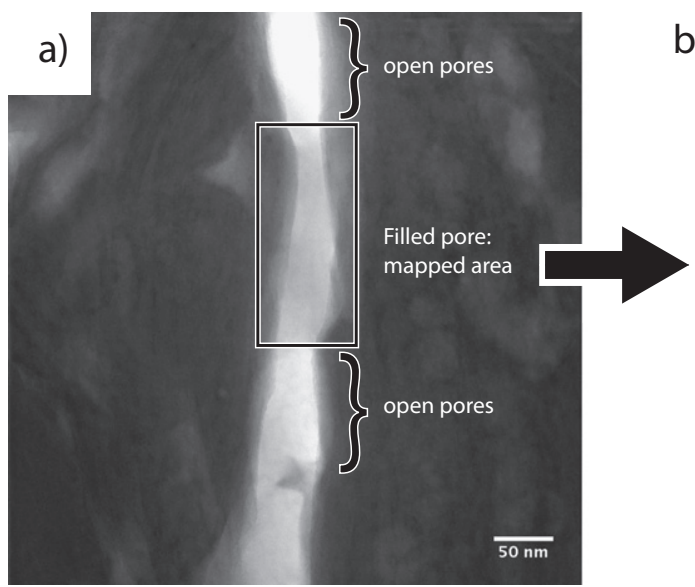

b)

Element distribution maps of pore fill

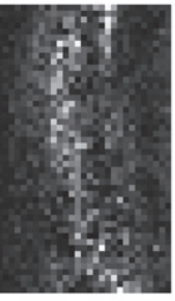

$\mathrm{Ca} / \mathrm{O}$

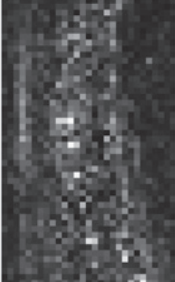

$\mathrm{Fe} / \mathrm{O}$

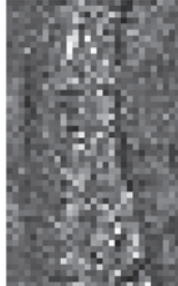

$\mathrm{Si} / \mathrm{O}$

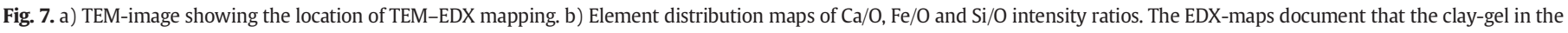
pore is enriched in $\mathrm{Ca}$, Fe, and $\mathrm{Si}$ when compared to the surrounding clay minerals.

that was developed by Valvatne and Blunt (2004). In order to use this simulator an equivalent pore network (i.e. skeleton) was extracted on the basis of the reconstructed voxel representation of the pore microstructure by using an implementation of the maximum ball algorithm developed by Dong and Blunt (2009). The extracted pore network was then used as input for the network simulator to predict the transport properties in block 3 . The predicted absolute permeability of the original pore space is on the order of $7 \times 10^{-19}\left[\mathrm{~m}^{2}\right]$. By setting $k_{0}$ to this value we are left with $\varepsilon_{c}$ as a remaining unknown parameter. The simulation predicts first air-flow through the microstructure at the water saturation $S=0.86$. Correspondingly, the smallest or critical air filled porosity that allows air flow through the pore space is given by $\varepsilon_{c} \sim 0.14 \phi$, which in the case of the sample from block 3 yields a value of 0.006 . Using these parameter values in Eq. (1) we can calculate air permeability as a function of porosity and for different values of water saturation. The calculations were done for relevant porosities that range between the "dry" percolation threshold along the z-direction (i.e. $\phi_{c}=0.02-0.03$ ) and the original porosity $(\phi<0.05)$ (Fig. 10). For a given water saturation, reduction of the pore space due to clay-gel formation leads to a drop in air permeability. This drop can be significant already for very small porosity changes, as shown by the steep slopes in the curves of Fig. 10. As expected, lower water saturation (i.e. higher air filled porosity) shifts the permeability and the associated drop of permeability towards lower porosities. Regarding the calculated gas permeabilities, which are not affected by precipitation (i.e. flat curve section in Fig. 14) there is surprisingly good agreement between calculations a)

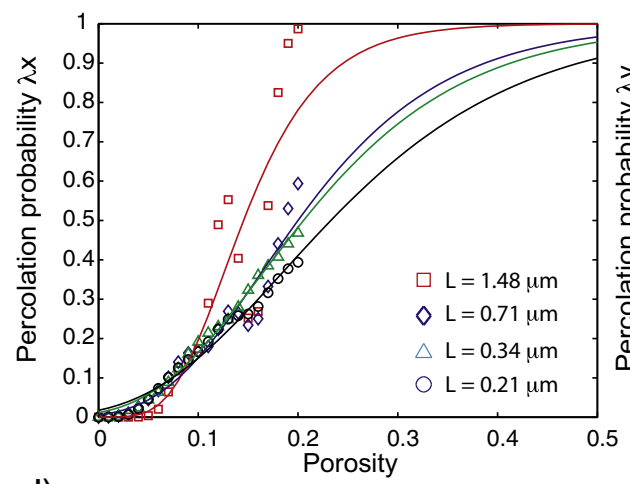

d)

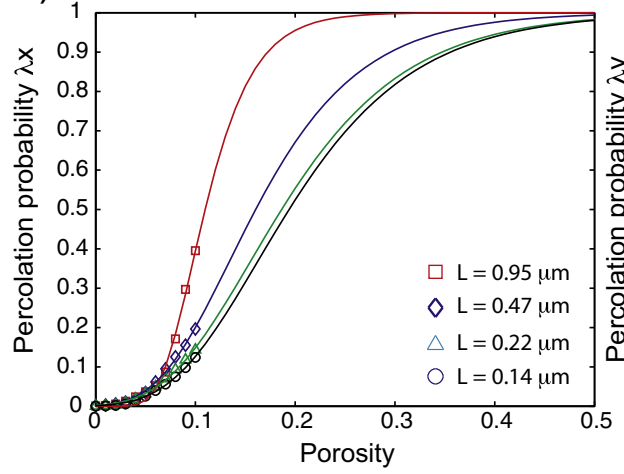

b)

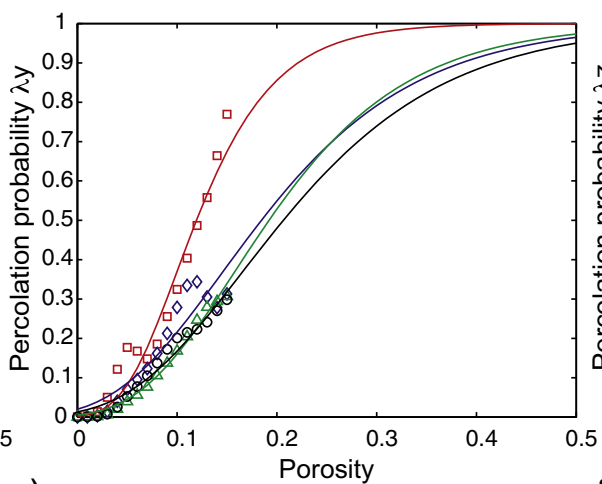

e)

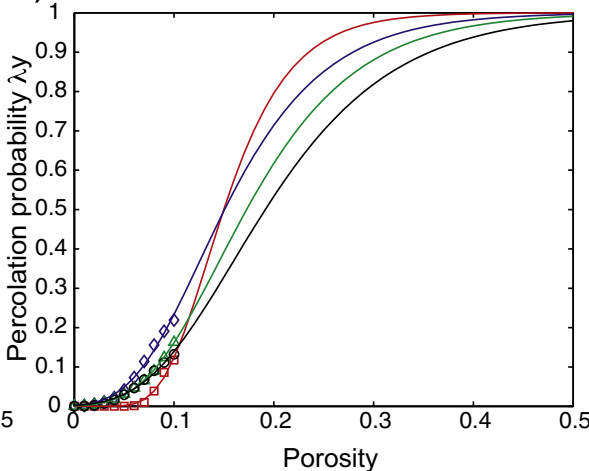

c)

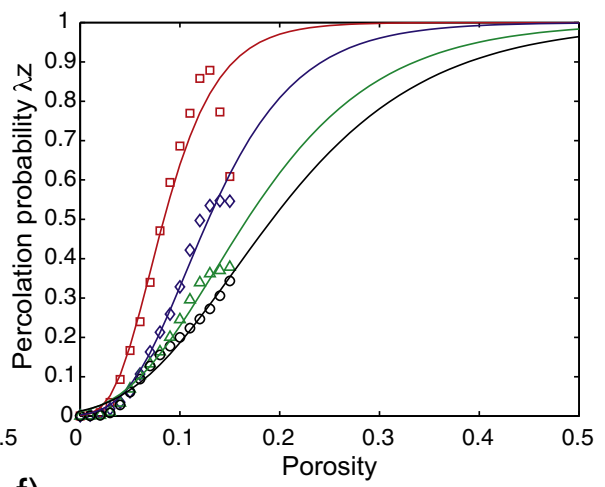

f)

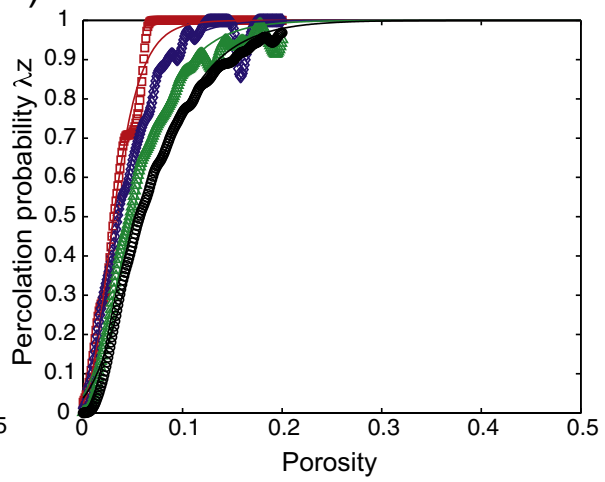

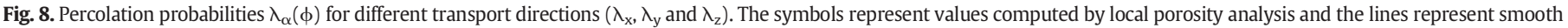

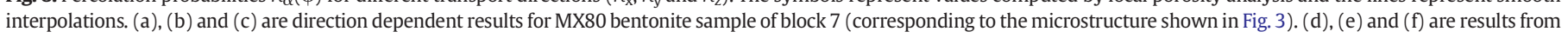
bentonite of block 3 (corresponding to Fig. 5). Note the different scale of the $\mathrm{x}$-axis in $\mathrm{f}$. 
a)

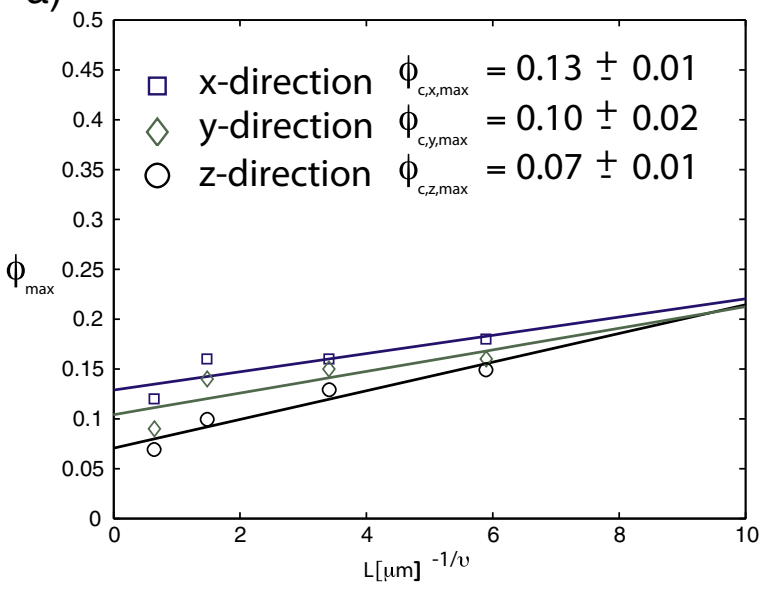

c)

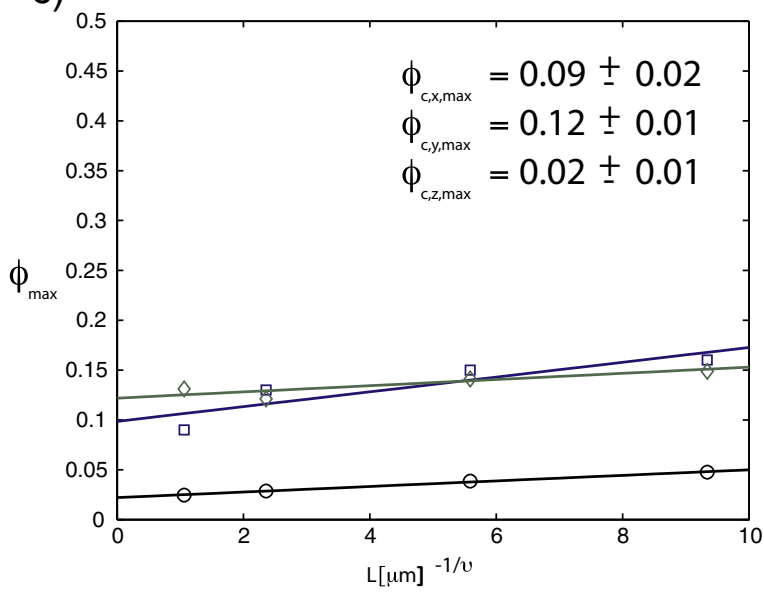

b)

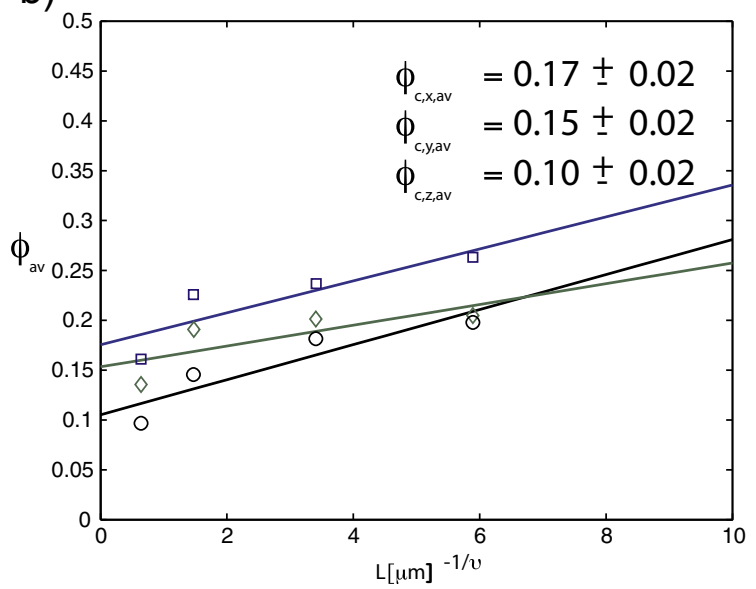

d)

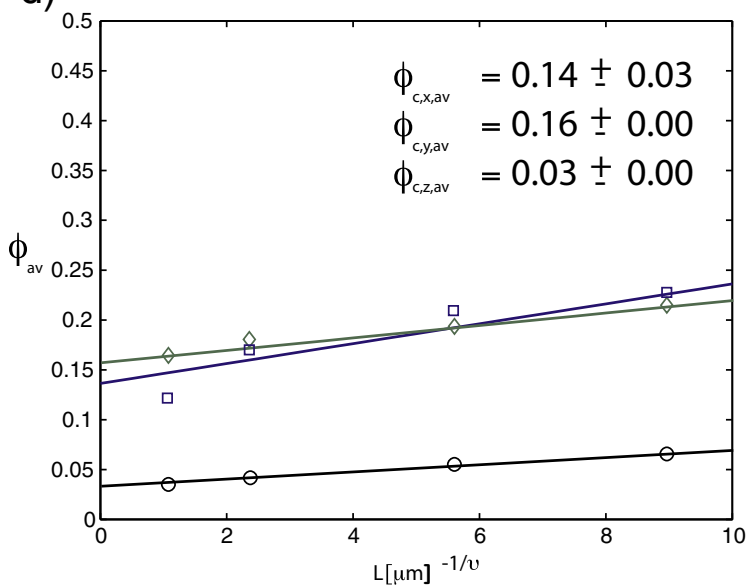

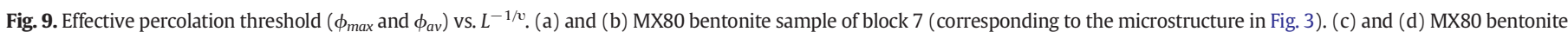

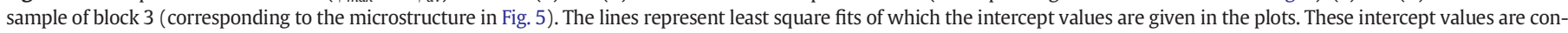
sidered as percolation thresholds. For both samples the percolation thresholds are clearly smaller in the $\mathrm{z}$-direction compared to the $\mathrm{x}$ - and $\mathrm{y}$-directions.

and experiment. For MX-80, gas flow experiments (Harrington and Horseman, 2003) yielded gas permeabilities in the order of $1 \times 10^{-20}$ $\left[\mathrm{m}^{2}\right]$ which is slightly higher than the calculated permeabilities at $\mathrm{S}=$

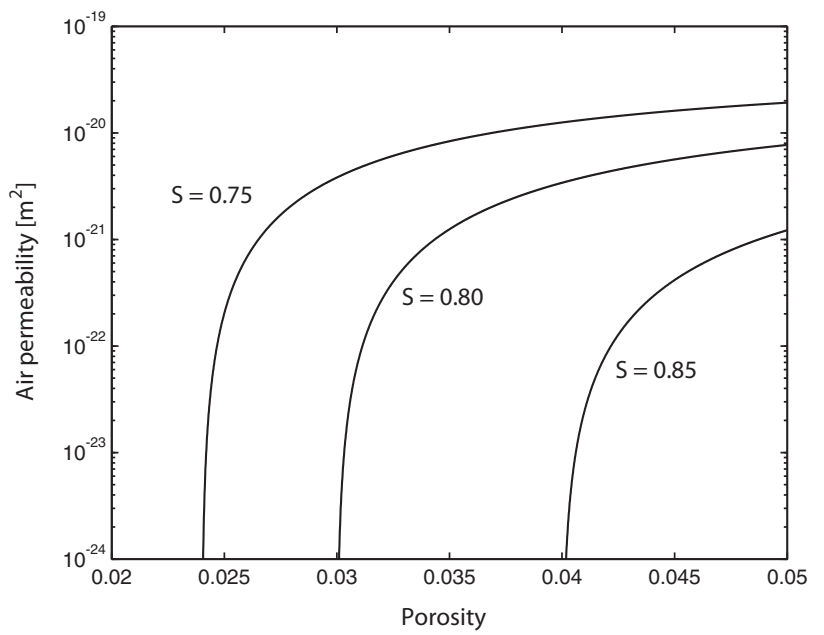

Fig. 10. Air permeability vs. porosity calculated for three different values of water saturation $\mathrm{S}$. The calculation is based on percolation-scaling and uses parameter values that were extracted by pore network modeling.
0.75. For higher water saturations, calculated gas permeabilities are roughly a magnitude lower when compared to experiments.

\subsection{Final remark}

It should be noted that the physical conditions imposed by the ABM experiment are not precisely that predicted from long-term simulations of the nearfield (i.e. buffer material around the waste) in radioactive waste deposits. It is expected that high temperatures during the early stages of the thermal pulse will inhibit full saturation of the buffer material such as MX- 80 bentonite. Based on simulations, it is predicted that this stage will last hundreds of years and is associated with a lack of water, which may limit clay-gel formation within intergranular pores. During later stages and under isothermal conditions the buffer material is expected to become fully saturated. Under such conditions the transport of dissolved species in compacted bentonite is expected to be diffusion controlled.

\section{Conclusions}

Based on FIB tomography and TEM imaging we present an insight into the evolution of the pore space of MX- 80 bentonite during the long-term ABM experiment. The study reveals that Fe released from corroding steel is redistributed in bentonite where it is used as a component for the formation of heavy minerals. During saturation, the initial intergranular pore space was filled with a clay-gel with properties 
similar to a viscous paste. The BSE intensity of the clay-gel suggests that the material is lower in density than the surrounding clay minerals, which leads to spatial density variations in almost fully saturated MX80 bentonite. Because FIB-nt is capable of resolving pores with radii $\geq 10 \mathrm{~nm}$, the percolation analysis provides information on potential transport along paths through these pores. Residual (i.e. open) intergranular porosity in all samples is clearly below the percolation threshold. During the early stages when saturation occurs, the initial porosity was higher and exceeded the percolation threshold albeit only in one direction and only in one sample (block 3). Thus, intergranular pore space was likely connected during the early stage of hydration but was subsequently filled by clay-gel that predictively reduced both porosity and connectivity.

\section{Acknowledgments}

This work was funded by the Swiss National Cooperative for the Disposal of Radioactive Waste (NAGRA). We thank an anonymous reviewer, whose comments and suggestions improved the quality of the work. We also acknowledge the contributions of Paul Wersin and Olivier Leupin, which were instrumental to our own thinking.

\section{References}

Bachmann, L., Mayer, E., 1987. Physics of water and ice: implications for cryofixations. In: Steinbrecht, R.A., Zierold, K. (Eds.), Cryotechniques in Biological Electron Microscopy. Springer, Berlin.

Bhuiyan, I., 2013. Microstructural Characterization of Iron Ore Green Pellets. (PhD Thesis) Department of Civil, Environmental and Natural Resources Engineering, Lulea University of Technology, Sweden.

Bunde, A., Havlin, S., 1995. Percolation I. In: Bunde, A., Havlin, S. (Eds.), Fractals and Disordered Systems. Springer, Berlin, pp. 59-114.

Clayton, C.J., Hay, S.J., 1994. Gas migration from accumulation to surface. Bull. Geol. Soc. Den. 41, 12-23.

Dong, H., Blunt, M.J., 2009. Pore-network extraction from micro-computerizedtomography images. Phys. Rev. E80, 036307.

Eng, A., Nilsson, U., Svensson, D., 2007. Alternative Buffer Material, Installation Report Aspo Hard Rock Laboratory (Report no. IPR-07-15).

Harrington, J.F., Horseman, S.T., 2003. Gas migration in KSB-3 buffer bentonite. Technical Report, TR-03-02. Swedish Nuclear Fuel and Waste Management Co.

Hilfer, R., 1991. Geometric and dielectric characterization of porous media. Phys. Rev. 44 $60-75$

Holzer, L., Indutnyi, F., Gasser, Ph., Münch, B., Wegmann, M., 2004. Three-dimensional analysis of porous BaTiO3 ceramics using FIB nanotomography. J. Microsc. 216, 84-95.

Holzer, L., Münch, B., Rizzi, M., Wepf, R., Marschall, P., Graule, T., 2010. 3D-microstructure analysis of hydrated bentonite with cryo-stabilized pore water. Appl. Clay Sci. 47, 330-342.
Hunt, A.G., 2005. Basic transport properties in natural porous media. Wiley Period. 10, 22-37.

Keller, L.M., Holzer, L., Wepf, R., Gasser, P., 2011. 3D geometry and topology of pore pathways in Opalinus Clay: implications for mass transport. Appl. Clay Sci. 52, 85-95.

Keller, L.M., Schuetz, P., Gasser, P., Holzer, L., 2013a. Pore-space relevant for gas permeability in Opalinus Clay: statistical analysis of homogeneity, percolation and representative volume element (RVE). J. Geophys. Res. 118, 1-14.

Keller, LM., Schuetz, P., Erni, R., Rossell, M.D., Lucas, F., Gasser, P., Holzer, L, 2013b. Characterization of multi-scale microstructural features in Opalinus Clay. Microporous Mesoporous Mater. 179, 83-94.

Keller, L.M., Seiphoori, A., Gasser, P., Lucas, F., Holzer, L., Ferrari, A., 2014. The pore structure of compacted and partly saturated MX-80 bentonite at different dry densities. Clays Clay Miner. 62, 174-187.

Liu, J., Neretnieks, I., 2006. Physical and chemical stability of the bentonite buffer. SKB report R-06-103. Swedish Nuclear Fuel and Waste Management, Stockholm, Sweden.

Marschall, P., Horseman, S., Gimmi, T., 2005. Characterisation of gas transport properties of the Opalinus Clay, potential host rock formation for radioactive waste disposal. Oil Gas Sci. Technol. 60, 121-139.

Milodowski, A.E., Cave, M.R., Kemp, S.J., Taylor, H., Green, K., Williams, C.L., Shaw, R.A. Gowing, C.J.B., Eatherinton, N.D., 2007. Mineralogical investigations of the interaction between iron corrosion products and bentonite from the NF-PRO Experiments (Phase 2). British Geological Survey Commissioned Report, CR/07/225 (76 pp.).

Münch, B., Holzer, L., 2008. Contraticting geometrical concepts in pore size analyzes attained with electron microscopy and mercury intrusion. J. Am. Ceram. Soc. 91, 4059-4067.

Otsu, N., 1979. A threshold selection method from gray-level histograms. IEEE Trans. Syst. Man Cybern. 9, 62-66.

Perdrial, J.N., Warr, L.N., 2011. Hydration behavior of MX80 bentonite in a confinedvolume-system: implications for backfill design. Clay Clay Miner. 59/6, 640-653.

Pringle, D.J., Miner, J.E., Eicken, H., Golden, K.M., 2009. Pore-space percolation in sea ice single crystals. J. Geophys. Res. 114, C12017.

Pusch, R., 2001. The microstructure of MX80 clay with respect to its bulk physical properties under different environmental conditions. Technical Report TR-01-08. Swedish Nuclear Fuel and Waste Management, Stockholm, Sweden.

Pusch, R., Karnland, O., Hoekmark, H., 1990. GMM - a general microstructural model for qualitative and quantitative studies of smectite calys. Technical Report TR-90-43. Swedish Nuclear Fuel and Waste Management, Stockholm, Sweden.

Stauffer, D., Aharony, A., 1995. Perkolationstheorie: Eine Einfuehrung. VCH, Weinheim, New York, Basel, Cambridge, Tokyo.

Svensson, D., Dueck, A., Nilsson, U., Olsson, S., Sanden, T., Lydmark, S., Jagerwall, S., Pederson, K., Hansen, S., 2011. Alternative buffer material, status of the ongoing laboratory investigation of reference materials and test package 1. Technical Report, TR-11-06. Swedish Nuclear Fuel and Waste Management Co.

Tomioka, S., Kozaki, T., Takamatsu, H., Noda, N., Nisiyama, S., Kozai, N., Suzuki, S., Sato, S., 2010. Analysis of microstructural images of dry and water-saturated compacted bentonite samples observed with X-ray micro CT. Appl. Clay Sci. 47, 65-71.

Vali, H., Hesse, R., 1992. The microstructure of dilute clay and humic acid suspensions revealed by freeze-fracture electron microscopy: discussion. Clay Clay Miner. 40, 620-623.

Valvatne, P.H., Blunt, M.J., 2004. Predictive pore-scale modeling of two-phase flow in mixed wet media. Water Resour. Res. 40, W07406.

Wersin, P., Jenni, A., Meader, U.K., 2013. Interaction of corroding iron with bentonite at repository conditions. Goldschmidt abstracts. Mineral. Mag. 77, 2482. 